\title{
Thin-layer agar (TL7H11) for rapid isolation of Mycobacterium tuberculosis in sputum specimens
}

\author{
Habiba Binte Alam¹, Md. Ruhul Amin Miah², Mostofa Kamal ${ }^{3}$, Chandan Kumar Roy ${ }^{2}$, Ahmed Abu Saleh ${ }^{2}$ \\ ${ }^{1}$ Department of Microbiology, Sir Salimullah Medical College, Dhaka, Bangladesh; ${ }^{2}$ Department of Microbiology and Immunology, Bangabandhu Sheikh Mujib Medical University, Dhaka, Bangladesh; \\ ${ }^{3}$ Department of Pathology and Microbiology, National Institute of Diseases of the Chest and Hospital, Mohakhali, Dhaka, Bangladesh.
}

\begin{abstract}
:
Background: Tuberculosis (TB) remains one of the major causes of death from a single infectious agent worldwide. The early detection of new cases of pulmonary tuberculosis is an important goal in tuberculosis control program. Objective: In this study, thin layer agar (TLA) culture was compared with Lowenstein-Jensen (LJ) culture for rapid detection of pulmonary tuberculosis. Methods: It was a cross sectional study conducted in National Tuberculosis Reference Laboratory (NTRL) of National Institute of Disease of Chest and Hospital (NIDCH), Dhaka, from July 2010 to June 2011. A total of 100 sputum smear positive for acid fast bacilli (AFB) by Z-N staining, pulmonary tuberculosis patients were included in this study. Samples were processed by modified Petroff method and then cultured on thin layer 7H11(TL7H11) plates and L-J tubes. TL7H11 plates were observed microscopically for microcolony growth once a week for 6 weeks, and L-J tubes were observed once a week for 8 weeks. Results: The recovery rates of mycobacteria on only TLA, only LJ and on both media were $90 \%, 97 \%$ and $88 \%$ respectively. Overall positivity was $99 \%$ in both L-J and TLA media. Mean time for detection of mycobacteria on TLA was $9.04 \pm 1.66$ days compared to $21.78 \pm 6.19$ days on L-J media. The rate of contamination was higher (6\%) in L-J media than in TLA media (4\%). Conclusion: The TL7H11 media can be used as an alternative to the Lowenstein-Jensen medium for early isolation of mycobacteria in resource constrained settings.
\end{abstract}

Key words: Mycobacterium tuberculosis culture, TL7H11 media, L-Jmedia.

[BSMMU J 2014; 7 (1) : 39-43]

\section{Introduction:}

Despite the discovery of the tubercle bacillus more than a hundred years ago, and all the advances in our knowledge of the disease made since then, tuberculosis still remains one of the major health problems facing mankind, particularly in developing countries. Presently, about one third of the world's population is infected with Mycobacterium tuberculosis. It is estimated that currently there are about 10 million new cases of tuberculosis every year with 3 million deaths occurring world-wide ${ }^{1}$. Currently, more people die of tuberculosis than from any other infectious disease. Death from tuberculosis comprises $25 \%$ of all avoidable deaths in developing countries. Nearly $95 \%$ of all tuberculosis cases and $98 \%$ of deaths due to tuberculo-

Address for Correspondence: Dr. Habiba Binte Alam Medical Officer Sir Salimullah Medical College, Dhaka, Bangladesh.

E-mail : koly_hasan@yahoo.com Mobile no-01716582563 sis are in developing countries and $75 \%$ of tuberculosis cases are in the economically productive age group ${ }^{2}$. Tuberculosis scenario of Bangladesh is not satisfactory at all as we stand in the 6 th position amongst 22 MTB burdened countries ${ }^{3}$. According to the WHO surveillance report 2009 the estimated annual incidence of all TB cases are 225 per 100,000 people. The prevalence of all cases is 425 and mortality is 51 per 100,000 people $^{4}$. The most apprehensive information is that about $50 \%$ adult population of Bangladesh are infected by Mycobacterium tuberculosis. Direct microscopy and Lowenstein-Jensen (L-J) medium are still considered the gold standard for the diagnosis of TB in low-income countries ${ }^{5}$. However, these methods have either low sensitivity, especially in paucibacillary pulmonary and extra-pulmonary TB, or take a longer time (3-4 weeks) for mycobacterial culture. Thinlayer agar and MODS (Microscopic observation broth drug susceptibility) are two promising modalities that 
detect the presence of mycobacteria by microscopic observation of colonies. Both the methods can detect the colonies microscopically within 1-2 weeks and can also give a presumptive identification of mycobacteria based on their characteristic colony morphology ${ }^{6,7}$.

The objective of the present study was to evaluate the TLA medium in terms of recovery rate, time for detection and contamination rate, comparing it with the Lowenstein-Jensen media.

\section{Methods:}

Ethical clearance was taken from Institutional review board of BSMMU. A cross sectional study conducted in National Tuberculosis Reference Laboratory (NTRL) of National Institute of Disease of Chest and Hospital (NIDCH), Dhaka, from July 2010 to June 2011. Clinically suspected pulmonary tuberculosis patients referred to the NTRL by physicians were enrolled for sputum examination. Patient positive for AFB by sputum smear Z-N stain were considered as pulmonary tuberculosis and were included in this study. A total of 100 patients of different age and different categories of treatment were enrolled in this study. Z-N staining was done on all the sputum samples and was processed for digestion and decontamination by modified Petroff method as described by WHO for TLA and L-J culture. A predesigned data collection sheet was used as research instrument.

TLA medium : The TLA technique uses Middlebrook $7 \mathrm{H} 11$ agar for the detection of microcolonies of various mycobacteria. About $6 \mathrm{ml}$ of the autoclaved $7 \mathrm{H} 11$ medium was poured into one out of 3 divided compartment of $96 \times 15 \mathrm{~mm}$ plastic petri dish, 10\% OADC (Oleic acid, Albumin, Dextrose, Catalase) and antibiotics, including Polymyxin B, Ticarcillin, Amphotericin and Trimethoprim, were added at concentrations of 200,000 unit/L, $100 \mathrm{mg} / \mathrm{L}, 10 \mathrm{mg} / \mathrm{L}$ respectively. A second set of $7 \mathrm{H} 11$ medium containing $500 \mu \mathrm{g} / \mathrm{ml}$ of Paranitrobenzoic acid (PNB) was prepared to differentiate between Mycobacterium tuberculosis complex and non-tuberculous mycobacteria (NTM); $0.1 \mathrm{ml}$ of the concentrated specimen was inoculated onto both TLA and PNB +TLA. All the plates were sealed with adhesive tape, leaving an area of about 2 $\mathrm{cm}$ for ventilation, and incubated at $37^{\circ} \mathrm{C}$ in a $5-8 \% \mathrm{CO} 2$ incubator for 6 weeks. The plates were examined for appearance of microcolonies (cording) once weekly by light microscopy. On appearance of visible colonies, colony morphology, rate of growth and pigment productions were studied. After that the growths were confirmed by acid fast staining with $\mathrm{Z}-\mathrm{N}$ method.

L-J medium: Three drops of pellet of each sputum sample after digestion and decontamination were inoculated into two slants of two set of Lowenstein-Jensen media using pasture pipettes. The inoculated media were incubated at $37^{\circ} \mathrm{C}$ in a slanted position with their screw caps loose for at least one day to ensure even distribution of inoculation. Then those were kept upright with caps tightened to minimize evaporation and drying of media. The bottles were examined after 72 hours to exclude contamination and if there was no contamination, the bottles were examined weekly up to 8 weeks ${ }^{8}$. If no growth observed after 8 weeks of incubation then the culture was reported as "no growth". In case of contaminated samples, result was interpreted from the other bottles. On appearance of visible colonies, colony morphology, rate of growth and pigment productions were studied. Mycobacterium tuberculosis produce dry, duff to cream colored rough surfaced colonies. The growths were confirmed by Z-N staining. After collection, data was checked for inadequacy, irrelevancy, and inconsistency. All data was analyzed with appropriate statistical stools and SPSS 15 program and presented as text, table and figure.

\section{Results:}

Out of 100 study population, $74(74 \%)$ were new cases and $26(26 \%)$ were re-treatment cases. A total of $99(99 \%)$ mycobacterial isolates (M.tuberculosis, $n=99$; NTM=0) were recovered from the $100 \mathrm{AFB}$ smear positive specimens. Rates of recovery of mycobacteria on TLA media and L-J media were $90 \%$ and $97 \%$ respectively. No growth was observed in 3 smear positive sputum in L-J media. Out of these 3 sputum samples, 2 specimens 
showed growth of mycobacterium in TLA media. Ten smear positive sputum samples yielded no growth in TLA media. Among them, 9 samples showed growth of mycobacterium in L-J media but one sample did not grow in L-J media. Culture positivity in both media was $88 \%$ (Tabel-I). Figure 1 shows a typical cord formation characteristic for M. tuberculosis on TLA. Figure 2 shows comparing time to growth detection among samples with different bacillary loads. The time for detection of positive cultures in TLA was 9.04 days which was faster than L-J 21.78 days $(\mathrm{p}<0.05)$. Rate of contamination observed in L-J media was $6 \%$ and in TLA media was $4 \%$.

Table-I

Growth of Mycobacterium tuberculosis in LJ and TLA media, duration of growth detection time and rate of contamination $(n=100)$.

\begin{tabular}{lccr}
\hline Name of & Culture & MTB isolation & Samples \\
the media & positive & time Mean & contamin \\
& $\mathrm{n}(\%)$ & ays \pm SD & ated $\mathrm{n}(\%)$ \\
\hline
\end{tabular}

$\begin{array}{lccc}\text { In TLA media } & 90(90) & 9.04( \pm 1.66) & 4(4) \\ \text { In LJ media } & 97(97) & 21.78( \pm 6.19) & 6(6)\end{array}$

Both TLA and $88(88)$

LJ media

Either in TLJ $99(99)$

A or L media

Note: MTB- Mycobacterium tuberculosis

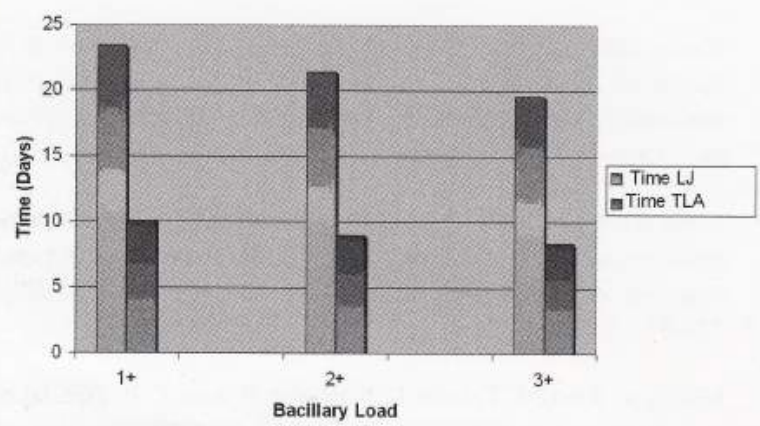

Fig-1: Microcolonies of Mycobacterium tuberculosis on TLA media observed under light microscope (objective 10x) at $10^{\text {th }}$ day in unstained petriplate

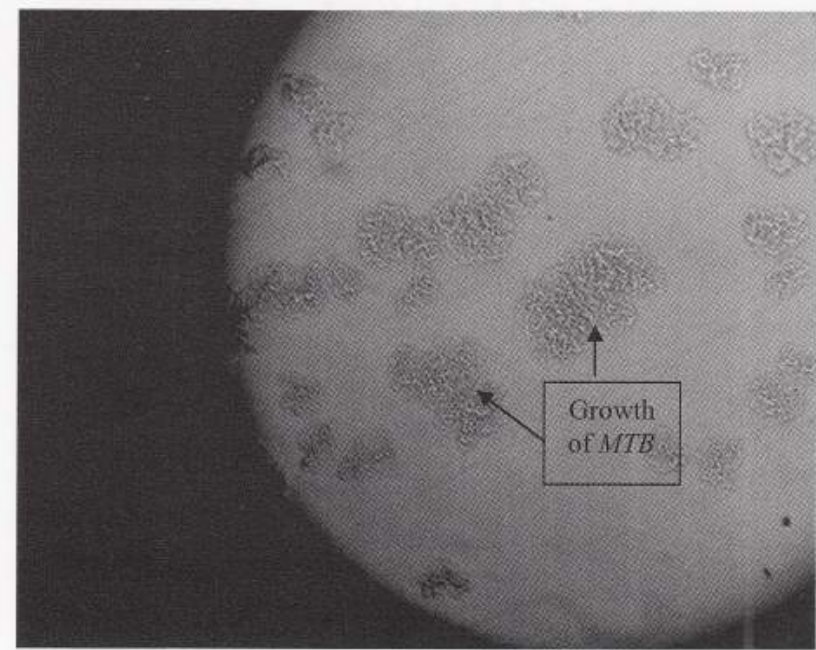

Fig-2: Mycobacterial growth detection time according to bacillary load of specimen.

\section{Discussion:}

Early diagnosis and prompt treatment have become a priority due to the rapid increase in the spread of TB world wide. Various studies have been carried out in various contexts to evaluate the performance of TLA ${ }^{9,10}$. Higher isolation rate of Mycobacterium tuberculosis was observed in L-J media in comparison with TLA media (97\% versus $90 \%)$ in this study. Combined isolation rate (either in TLA or in L-J media) was 99\%. Similar finding was observed in a study of America in 1993 and in Colombia in $2004^{11,12}$. In their studies isolation rate of MTB in TLA media was $83 \%$ and $74 \%$ and in L-J media was $93 \%$ and $91 \%$ respectively. On the contrary another study in Spain by Idigoras et al (1995), overall isolation rate of MTB in TLA and L-J media was $90 \%$ and $77 \%$ respectively ${ }^{13}$. In house preparation of TLA medium rather than obtained from commercial source and incubating in $5 \% \mathrm{CO}_{2}$ might be the reasons for higher isolation rate of MTB in TLA media. In this study, it was observed that in average after 20 days of incubation, TLA media became dry and shrunken and sometimes cracked. Some relatively slow growing mycobacteria might be missed for these reasons in TLA media.

In present study, TLA showed statistically significant shorter growth detection time than L-J media (9.04 days versus 21.78 days). Mean growth detection time in TLA 
media was 11 days and in L-J media was 23 days had also been reported by Welch et al (1993) in America ${ }^{11}$. It was not found any study contrary to this study, regarding growth detection time. Shorter growth detection time of $M T B$ in TLA media might be due to growth supplements such as Oleic acid, Albumin, Dextrose and catalase (OADC), varieties of inorganic salts, glycerol and $\mathrm{CO}_{2}$ containing atmosphere.

Mean growth detection time for $M T B$ varied depending on the bacillary load in sputum in both TLA and L-J media. But the variation of time was statistically not significant in the present study. Robledo et al (2006) in Latin America, showed similar finding ${ }^{6}$. They found mean growth detection time of MTB in grade $1+, 2+$ and $3+$ specimen in TLA media was 8 days, 7.5 days and 7 days respectively. In L-J media mean growth detection time was 28 days, 22 days and 21 days respectively. Relatively shorter mean growth detection time in higher bacillary load is natural and need not to be explained.

In present study rate of contamination in L-J media was $6 \%$ and in TLA media was $4 \%$. However, Martin with his associates (2009) revealed that, the contamination rate was $16 \%$ and $26 \%$ respectively in TLA media ${ }^{14}$. Contamination rate was much higher in these studies than this one. The probable cause may be that in these studies they used Trimethoprim, Amphotericin and Piperacillin antibiotics (TAP) in TLA media for prevention of contamination. Due to unavailability of TAP preparation in Bangladesh in present study Polymyxin B, Ticarcillin, Amphotericin, Trimethoprim (MAST selectatab) were used. Before introducing MAST selectatab a pilot study was carried out to evaluate its inhibitory effects on the growth of Mycobacterium tuberculosis and its inhibitory effects on contaminating organisms.. All the samples were processed freshly within 24 hours after collection, to minimize contamination.

\section{Conclusion:}

TLA media was evaluated in this study for isolation of MTB. Results of this study revealed that TLA media is a reliable alternative to L-J media for the rapid isolation of M.tuberculosis . Rate of contamination was also low in
TLA media and it required no additional equipments or materials. The simplicity of this one-plate format may favor its implementation and applicability in mycobacterial diagnostic laboratories, especially in resource limited countries like Bangladesh.

\section{Acknowledgement:}

The authors express their gratitude to the NTRL of NIDCH for giving opportunity to conduct this research in their Laboratory. We are also grateful to Dr. Shaheda Anwar for statistical analysis.

\section{References:}

1. Dye C, Scheele S, Pathania V, Raviglione MC. Global burden of tuberculosis: estimated incidence, prevalence and mortality by country. JAMA 1999; 282: 677.

2. World Health Organization. Global tuberculosis Control, Surveillance, Planning, Financing. Online [Internet].2002 [cited 2011 Feb 7]: Available from: WHO/CDS/Tuberculosis/2002.

3. National Tuberculosis programme, National tuberculosis control in Bangladesh, Annual report 2009, pp: 2-5. Online [Internet].2009 [cited 2011 April 11]: Available from: www.ntpban.org.2009.

4. World Health Organization. Multidrug and extensively drug-resistant TB (M/XDR-TB): 2010 global report on surveillance and response, pp: 6-7. Online [Internet].2010 [cited 2011 July 6]: Available from: WHO/HTM/TB/2010.

5. Lutfor $\mathrm{AB}$. Extensively drug resistant tuberculosis (XDR-TB). Bang. J. Med Microbiol 2009; 03 (01): 1-3

6. Robledo J, Mejia GI, Morcillo N. Evaluation of a rapid culture method for tuberculosis diagnosis: a Latin-American multicentre study. Int J. Tuberc Lung Dis 2006; 10 (6); 613-619.

7. Giampaglia CMS, Martins MC, Inumaru VTG, Butuem IV,Telles MAS. Evaluation of a rapid differentiation test for the Mycobacterium tuberculosis complex by selective inhibition with p-nitrobenzoic acid and thiophene-2-carboxylic acid hydrazide. Int J Tuberc Lung Dis 2005; 9: 206-209.

8. Kantor IND, Kim SJ, Frieden T, Laszlo A, Luelmo F, Norval P, Rieder $\mathrm{H}$, Valenzuela $\mathrm{P}$ and Weyer $\mathrm{K}$. Laboratory service in tuberculosis control, Geneva, World Health Organization; 1998. pp: 7 (part-1), 15-17 (part-II), 37-71 (part-III).

9. Mejia GI, Castrillon L, Trujillo $\mathrm{H}$ and Robledo JA. Microcolony detection in $7 \mathrm{H} 11$ thin layer culture is an alternative for rapid diagnosis of tuberculosis. Int. J. Tuberc Lung Dis 1999; 3(2): $138-42$.

10. Martin A, Krista F, Francis V, Francoise P, Juan C P. Thin layer agar compared to BACTEC MGIT 960 for early detection of Mycobacterium tuberculosis. J Microbiol Methods 2009; 78: 107-108. 
11. Welch DF, Guruswamy AP, Sides SJ, Shaw $\mathrm{CH}$ and Gilchrist $\mathrm{M}$ J. Timely culture for mycobacteria which utilizes a microcolony method. J. Clin. Microbiol 1993; 31(8): 2178-2184.

12. Mejia GI, Guzman A, Agudelo CA, Trujillo H and Robledo J. Five years experience with thin layer agar for the rapid diagnosis of tuberculosis. J. Biomed 2004; 24 (1): 52-9.

13. Idigoras $\mathrm{P}$, Trallero $\mathrm{E} \mathrm{P}$, Alcorta $\mathrm{M}$, Gutierrez $\mathrm{C}$ and Baroja I M. Rapid detection of tuberculous and non-tuberculous mycobacteria by microscopic observation of growth on Middlebrook 7H11 agar. Eur. J. Clin. Microbiol. Infect. Dis. 1995; 14(1): 6-10.

14. Martin A, Waweru PM, Okatch FB, Ouma NA, Bonte L, Varaine
15. F and Portaels F. Implementation of the Thin Layer Agar Method for diagnosis of smear-negative Pulmonary Tuberculosis in a setting with a high Prevalence of Human Immunodeficiency Virus infection in Homa Bay, Kenya. J. Clin. Microbiol 2009; 47(8): 2632-34.

16. Mejia GI, Castrillon L, Trujillo $\mathrm{H}$ and Robledo JA. Microcolony detection in 7H11 thin layer culture is an alternative for rapid diagnosis of tuberculosis. Int. J. Tuberc Lung Dis 1999; 3(2): $138-42$.

17. Khaled NA and Enarson DA. Tuberculosis. A Manual for Medical Students. Geneva, World Health Organization. 1999; p: 14-21 \& 95-99. 